\title{
Rapid acquisition method of sea vessel radiating transient noise effective time based on the power-law
}

\author{
Tongkui Yu, 2, a, Caogen Xiong ${ }^{2, b}$, Xiubo Wang ${ }^{2, c}$, Xuefeng Dai ${ }^{2, d}$ and \\ Tongjie Qin ${ }^{2}$ e
}

${ }^{1}$ College of Underwater Acoustic Engineering Harbin Engineering University, Harbin 115000, China

${ }^{2}$ Dalian Scientific Test and Control Technology Institute, Dalian 116013, China

aytk2222@163.com, bXiong caogen@163.com, cwangxiubo@163.com, ddxf2010@163.com, eqingtongjie@163.com

Keywords: ships, transient noise, power-law, significant moment, wavelet transform.

\begin{abstract}
Ship transient noise generally has the characteristics of strong energy, short duration, and frequent appearance and so on, for sonar, these features not only affect their own working performance, but also are easily detected by enemy sonar, so, grasping the features and magnitude of underwater radiation noise will be conducive to the management work of guiding ship radiating transient noise. In order to effectively evaluate the acoustic features and magnitude of ship transient noise, accurately acquiring the beginning and end time is needed, some traditional transient signal detection methods can realize the extract of central moment, but the accuracy of extracting persistent time is poor, based on this reason, the paper uses power-law algorithm for transient signal waveform of the structure of the "sensitivity", combines with line-spectrum extraction technology and wavelet transform, gives full play to the advantages of the algorithm itself, to realizes rapid intercept of effective analysis time of ship transient noise, thus laying the foundation for characteristic analysis and level assessment of transient noise. The theoretical simulation result shows that this method can effectively eliminate the interference of steady state and background noise, and ensures the extraction of effective analysis time to transient noise. By conducting sea sound simulation experiment, using this method to analysis underwater radiation noise data, and the analysis result shows that this method can quickly and efficiently extract the beginning and end time of transient state noise ,obtains the acoustic features and magnitude, and has a better engineering application value.
\end{abstract}

\section{Introduction}

The radiation noise of a seagoing vessel is mainly produced by weapon emission, steering and so on, generally has characteristics of strong energy, short duration, frequent appearance, etc. For sonar, these features not only affect their own sonar performance, but also easily detected by enemy sonar. At the same time, both sonar detection or vibration and noise reduction, are carried out around the acoustic characteristics of ship, so, it is very important that how to accurately obtain the order of ship noise transient and acoustic features from the measured data.

Ship transient noise belongs to non-steady state noise, in order to analysis it accurately , the testing data of ship transient noise must be first detected to gain the beginning and end time, and then cut out transient noise from measured data, so, the accuracy of transient noise data capture will directly affect magnitude calculation results. For this, a large number of studies have been made in domestic and overseas, and the detection methods of narrow-band [1], Power-law [2], local instantaneous energy density [3] and higher-order statistics [4-5] etc are put forward, these methods have their own scope of application and advantages, most are limited to the maximum peak transient noise time for testing, and unable to provide the beginning and end time. For this reason, this article is based on the power-law algorithm for transient signal waveform of the structure of the "sensitivity", combining the methods of line-spectrum extraction technology, wavelet transform, and second order partial derivative function, to extract the beginning and end noise, theoretical simulation and experimental study show that the 
algorithm can accurately obtain transient state noise time effectively, get ship scale and characteristics of the transient state noise spectrum, and have good engineering application value.

\section{Theoretical Analysis}

\subsection{Nuttall's Power-Law Statistic}

Nuttall [6-7] firstly proposed the Power-law detector. When sequence is Gaussian white noise, the square of discrete Fourier transform magnitude is independent distribution exponential random variables, and there is no signal exist at this time After the signal appears, time series of DFT square amplitude value no longer obey exponential distribution, and distinguish signal is exist. Nuttal puts forward the basic assumption as:

$$
\begin{aligned}
& H_{0}: \quad f(x)=\prod_{k=1}^{N} \frac{1}{\mu_{0}} e^{-\frac{X_{k}}{\mu_{0}}} \mu\left(X_{k}\right) \\
& H_{1}: \quad f(x)=\prod_{k \in S}^{N} \frac{1}{\mu_{1}} e^{-\frac{X_{k}}{\mu_{1}}} \mu\left(X_{k}\right) \bullet \prod_{k \in S}^{N} \frac{1}{\mu_{0}} e^{-\frac{X_{k}}{\mu_{0}}} \mu\left(X_{k}\right)
\end{aligned}
$$

where $N$ is Analysis points, $X$ is the square amplitude of Fourier transform result of time series, ${ }^{\mu}$ is step function, $S$ is transient signal, and $M$ is the length.

The equation's basic idea can be expressed as: When there is signal, the size of the 2 data points distributed evenly over three Fourier transform, in the case of $H_{1}$ established, accurate probability distribution function depends on the transient signal itself. Due to the method is sensitive to the characteristics of transient noise such as beginning time, signal waveform structure and duration time, so, foreign scholars widely use it into underwater acoustic transient signal discrimination.

Using the power-law algorithm analysis transient signal, and comparing discriminant results with given information, as shown in figure 1,from figure 1 we can see that transient noise is Gaussian amplitude modulation signal, central moment is $2 \mathrm{~s}$, short duration is about $0.4 \mathrm{~s}$, this algorithm can well reflect the transient signal the beginning and end time.

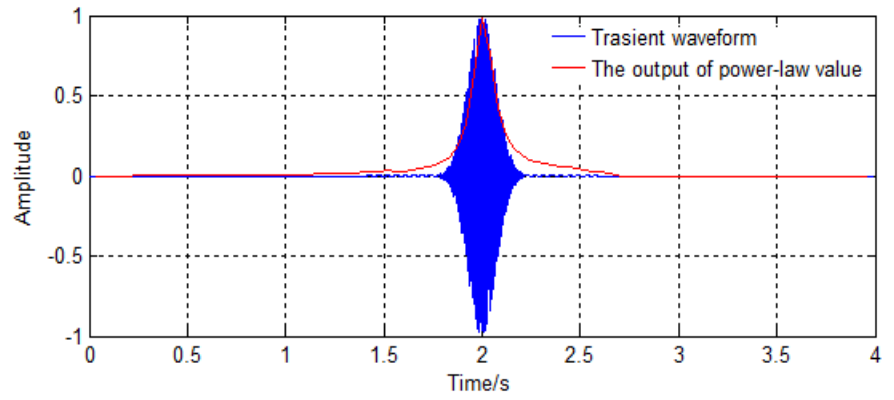

Fig. 1.Power-law discriminant algorithm results

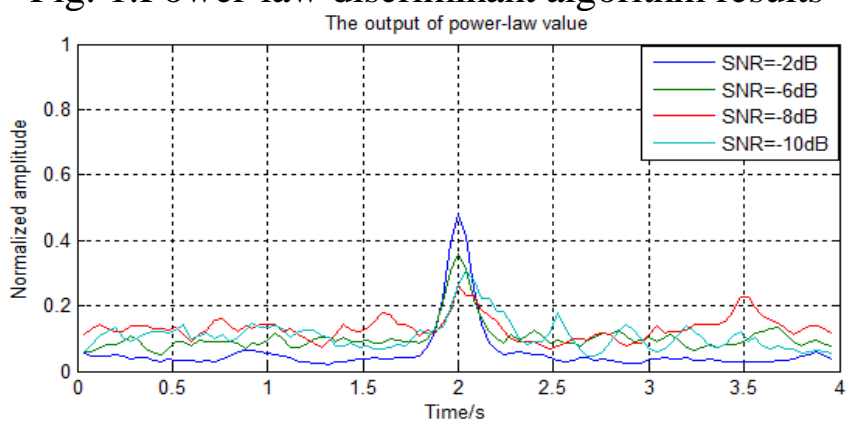

Fig. 2.Discrimination results under the condition of different instantaneous signal-to-noise ratio

\subsection{Line spectrum extraction and wavelet denoising}

For the ship transient noise data, there are many interference signal, these signals will directly affect the extraction accuracy of transient noise analysis period of time, and noise is also bad for the characteristics of the transient signal analysis, there is a certain impact on the timing of the operation at the same time, therefore, before to the measured data processing, to eliminate the steady state signals properly and the interference of background noise is very necessary, this paper uses the line-spectrum 
extraction technology and wavelet transform to eliminate pattern and the interference of background noise respectively.

Assuming that ship transient noise measured data is $y(t)$, Mainly overlay formed by static noise $x(t)$, transient noise $s(t)$ and interfering noise.

$$
y(t)=x(t)+s(t)+e \cdot n(t)
$$

For steady-state signal $x(t)$ can be regarded as a linear combination of the periodic signal, Fourier transform to get the $X\left(e^{j \omega}\right)$ is called $X(t)$ of spectrum.

$$
\begin{aligned}
& X\left(e^{j \omega}\right)=\int X(t) e^{-j \omega t} d t \\
& X(t)=\frac{1}{2 \pi} \int_{2 \pi} X(j \omega) e^{j \omega t} d \omega
\end{aligned}
$$

Using the theory of Fourier transform reversibility to make fourier transform of signal, extracting the characteristic spectrum, and then to inverse discrete Fourier transform, so as to realize the effect of line-spectrum extraction, also applying to the certain bandwidth of frequency spectrum.

On this basis, taking advantage of the theory of wavelet transform in signal noise reduction processing, usually adopting Mallet [8-9] fast algorithm for signal wavelet decomposition, if $f_{k}$ is the signal of discrete sampling data, $c_{0, k}=f_{k}$, Multi-resolution analysis formula with a signal are:

$$
\begin{aligned}
& c_{j+1, m}=\sum_{k} \bar{h}(k-2 m) c_{j, k} \\
& d_{j+1, m}=\sum_{k} \bar{g}(k-2 m) c_{j, k}
\end{aligned}
$$

Where $c_{j, k}$ is the signal approximation coefficients, and $d_{j, k}$ is detail coefficients of signals.

Wavelet multi-resolution analysis is the signal $y(t)$ which analyzed by different temporal resolution draw together step by step, projects a known function $y(t)$ to orthogonal function space. The same scale wavelet function or scaling function constitute the base of a function space, and the base scale of different function space is different, and represents different frequency characteristic. By constantly projection, the component of primitive function in the random subspace can be obtained gradually, realizing signal analysis by the course and fine. Here the signal is divided into approximate part and detail part, which iscalled approximation in part refers to the signal can be projected onto a large scale low frequency into the amount of space, while the detail refers to the signal with small scale space corresponding to high frequency component. Signal reconstruction formula is:

$$
c_{j, k}=\sum_{m} h(k-2 m) c_{j+1, m}+\sum_{m} g(k-2 m) d_{j+1, m}
$$

The measured data $y(t)$ after eliminating static noise signal model is:

$$
y(t)=s(t)+e \cdot n(t)
$$

Making soft threshold processing after discrete wavelet transform of $y(t)$ :

$$
\bar{S}=T(Y, t)=\left\{\begin{array}{cc}
\operatorname{sgn}(Y)(|Y|-t) & |Y| \geq t \\
0 & |Y|<t
\end{array}\right.
$$

In the type, $\bar{S}$ is the estimation of $S, t=e \sqrt{2 \log (N) / N}, N$ is the length of the signal. Making inverse transformation for $\bar{S}$ can get the transient signal after demising [10].

\section{Simulation}

The simulation example: Signal is linear superposition of transient and steady state signal, signal length is four seconds, in the steady state noise is $20 \mathrm{~Hz}$ and $100 \mathrm{~Hz}$ sinusoidal signal, which is $100 \mathrm{~Hz}$ gaussian amplitude modulation signal, center point is two seconds, pulse width is 0.4 seconds, introducing the gaussian white noise at the same time, instantaneous signal to noise ratio $-15 \mathrm{~dB}$, the time domain waveform is shown in figure 3. 

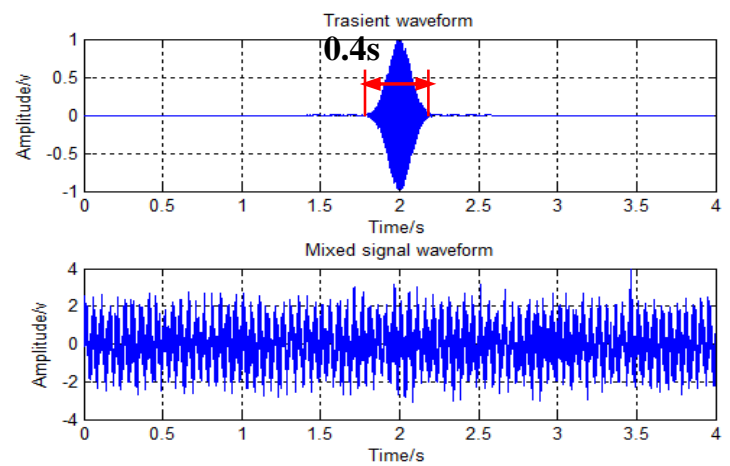

Fig. 3. The simulation signal time domain waveform

Making use of short time Fourier transform to calculating results, as shown in figure 4, from which we can see that, there is two single frequency pattern, near the 2 s exist transient signal, both duration and characteristic frequency have a certain deviation with simulation information, which reason is that the time - frequency resolution of short time Fourier transform is relatively low.

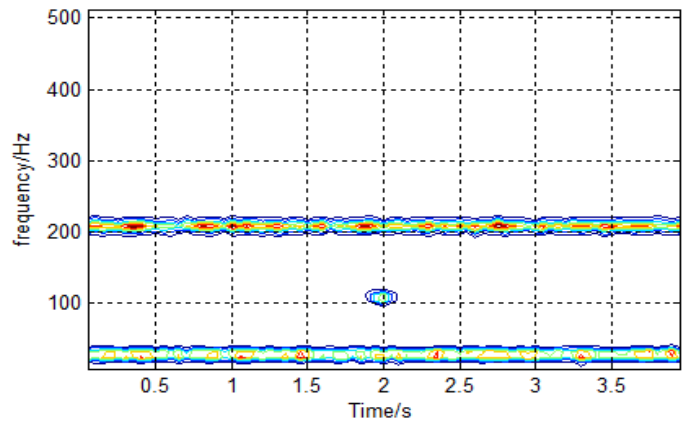

Fig. 4. STFT time-frequency diagram

In order to compare the extraction of accurate transient signal the beginning and end of time, using the power-law under the algorithm for the sensitivity of the transient signal analysis results are given, As shown in figure 5.From the figure we can see that when the instantaneous signal-to-noise of transient signal is bad, the power-law algorithm cannot correctly judging the results of the transient signal is given.

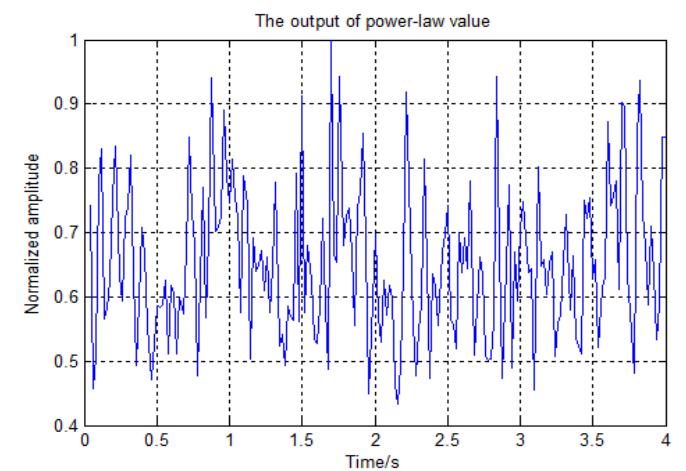

Fig. 5. The Power-law discriminant result

From the time-frequency analysis result we can know that mixed signal has two steady state signals, then using the line-spectrum extraction technology to eliminate steady state signal from signals to be analyzed, as shown in figure $6,25 \mathrm{~Hz}$ and $200 \mathrm{~Hz}$ characteristic line disappear. 

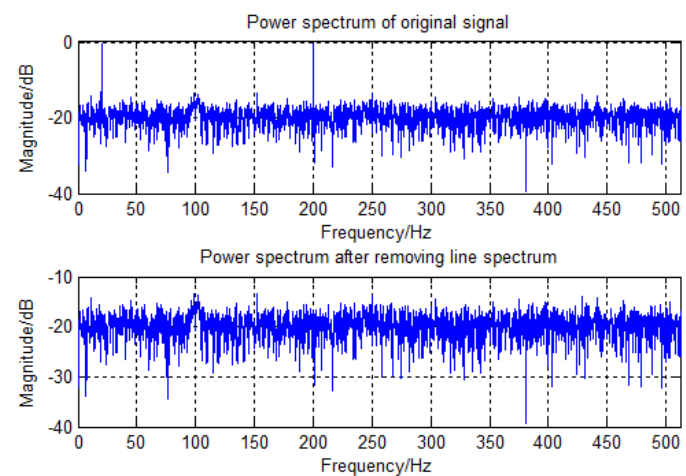

Fig. 6 The contrast figure before and after the steady state noise are eliminated

On the basis of above analysis, using the power-law algorithm to a further analyze, concrete analysis results are shown in figure 7, From the figure we can see that the power-law algorithm discriminant results appear "envelope" in the $2 \mathrm{~s}$, so center point is $2 \mathrm{~s}$, corresponding to the last time about $1.8 \mathrm{~s}$ to $2.2 \mathrm{~s}$, which is completely consistent with artificial intelligence.

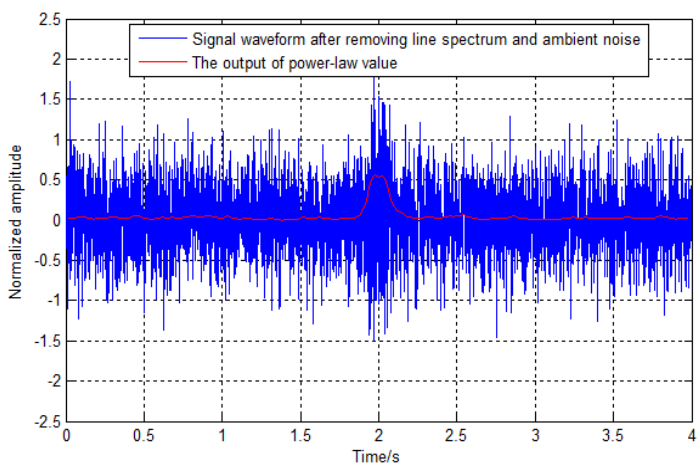

Fig. 7 The contrast figure of time domain waveform after the removal of line-spectrum and the power-law discriminant result.

From what has been discussed above, using the power-law algorithm for the transient energy and the structure of the waveform signal sensitivity, combining line-spectrum excluding with wavelet denoising technique, can realize the discrimination of transient state noise in the production and end theoretically.

In order to verify the feasibility and effectiveness of the algorithm in engineering application, further sourcing simulation test, details are as follows.

\section{Experimental Validation}

Experimentproceed on sea, using fore and aft ship well scalable hangers install the transmitting transducer and hydrophone, respectively(as shown in figure 8), choosing to carry out the sound transmitting and receiving test in the quiet night under the Marine environment. On the condition that emission source frequency, amplitude and phase are known, acquiring the acoustic radiation information in aqueous medium synchronously, using the transient state noise information processing method established above to analyze, so as to achieve the purpose of the verification algorithm.

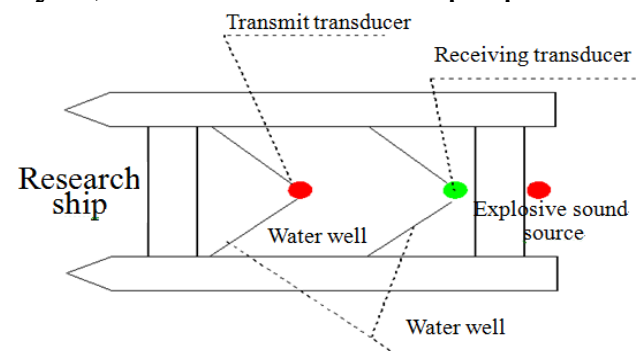

Figure 8.Schematic diagram of experiment 
Testing system mainly includes launch and receiving system, launch system is mainly composed of signal source, power amplifier, and transmitting transducer, the receiving system is mainly composed of hydrophone, signal conditioning, collector and laptop computers.

The test explosion sound source is used to simulate impact signal, uses transducer send steady state noise which amplitude, frequency and phase are known, radiation noise sampling frequency is 100 $\mathrm{KHZ}$, test process to ensure complete test data record.

Figure 9 and 10 respectively give the underwater radiation noise figure and the time domain waveform $1 / 3$ octave spectrum diagram, from which we can see that impact signal exist in the vicinity of 0.5 embodied as broadband spectrum on frequency spectrum, and there is $100 \mathrm{~Hz}$ characteristic spectrum.

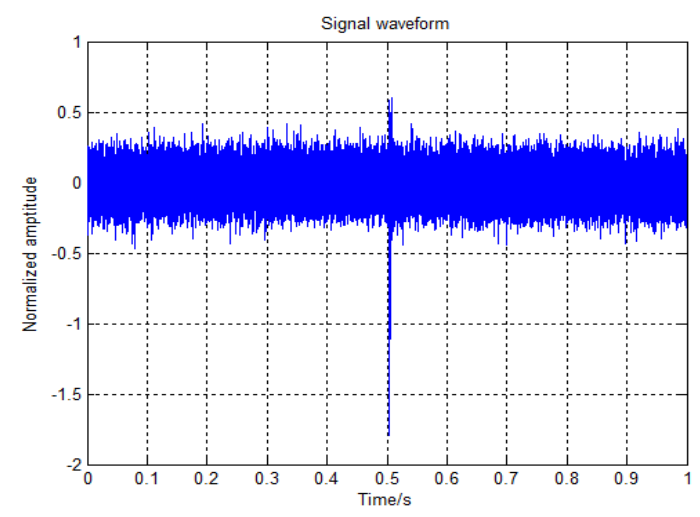

Figure 9. Radiation noise figure the time domain waveform

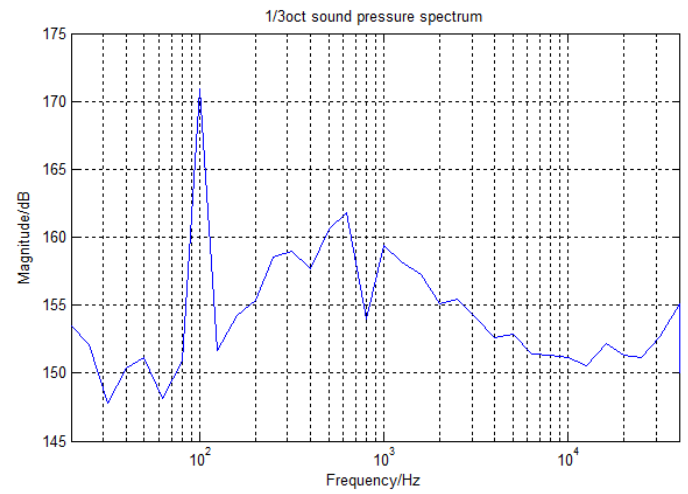

Figure 10 Radiation noise $1 / 3$ oct spectrum diagram

Using the informationprocessingmethodestablished in the text, to imitate rejecting and radiated noise data of denoising processing, specific analysis of the results as shown in figure 11 and figure 12.From the figures we can see that $100 \mathrm{~Hz}$ characteristic spectrum disappeared, after removal of line-spectrum and Marine environment noise, the transient state noise is very obvious.
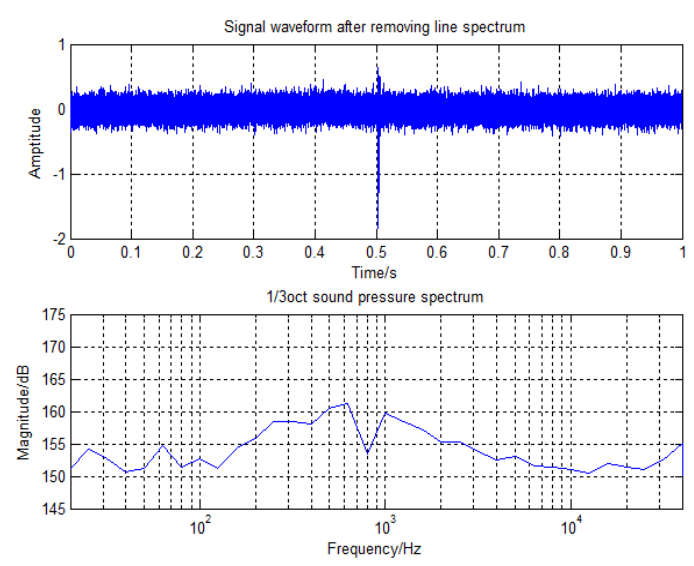

Figure11. Signal time domain and spectrum diagram after radiation noise pattern is put forward 


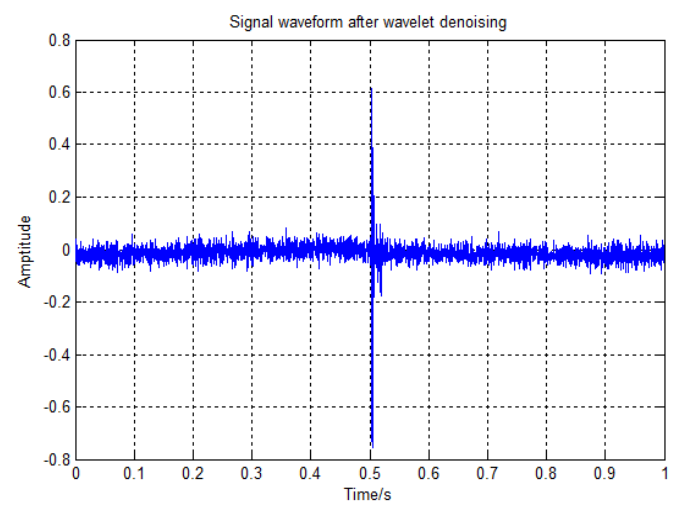

Figure 12 Signal time domain waveform figure after radiation noise to remove interference

On the basis of above analysis, using the power-law algorithm to detect transient signal generated and end, as shown in figure 13,0.515s is the center of the instantaneous signal, by the second order partial derivative function value we can know that In $0.5076 \mathrm{~s}$ and $0.5228 \mathrm{~s}$ curve jump phenomenon exists, respectively correspond the production and end of transient noise.
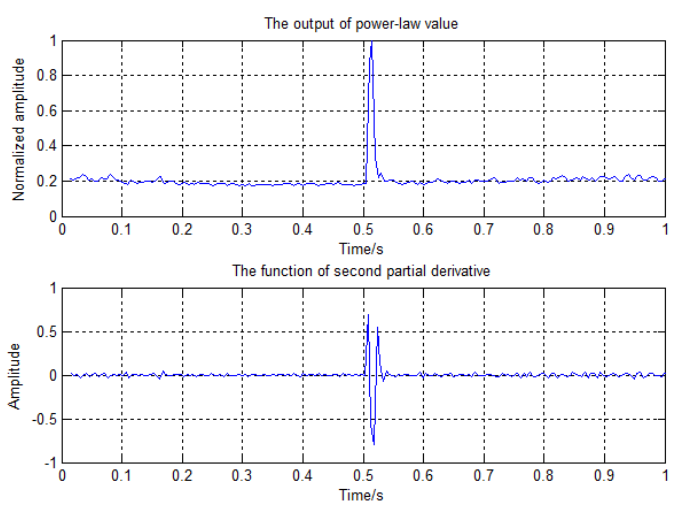

Figure13 the transient state noise effective moment discriminant analysis

On the basis of the transient signal energy calculation method, the total sound level of transient noise can be get as:

$$
S_{a l l}=10 \log _{10}\left[\int_{0}^{T} p^{2}(t) d t-\int_{0}^{T} p_{x}^{2}(t) d t-\int_{T_{n}}^{T_{n}+T} p_{n}^{2}(t) d t\right]+L M D+20 \lg D=172.1 d B
$$

Among this, $p(t), p_{x}(t)$ and $p_{n}(t)$ respectively are momentary signal, steady noise and marine background noise sound pressure, $L M D$ is the sensitivity of hydrophone, $D$ is the distance between the sound source hydrophone to explosion.

\section{Conclusion}

Through the study of transient noise a variety of information processing method, especially for the data analysis of source validation test data on the condition of sea anchor method, we can obtain the following conclusions:

(1) The discriminated method of effective analysis moment of transient noise which is based on power-law algorithm, not only can detect the transient noise at the center of the moment, but also is sensitive to the characteristics such as the production and the end, the structure of the signal waveform, and can be used to get the ship and end point of the transient noise;

(2) Line-spectrum extraction and wavelet denoising technique based on Fourier transform can effectively remove the steady state noise and the interference of environmental noise to transient state noise;

(3) Theoretical simulation and experimental results indicate that: The access method of effective analysis moment of transient noise which is based on power-law algorithm, can be well received the beginning and end time of ship transient state noise, laythefoundationof accurate calculation of the transient noise sound level, and has good engineering application value. 


\section{References}

[1]. P.Kirsteins, J.Fay. Suppressing reverberation by multipath separation for improved buried objuect detection. IEEE Oceans, 2001, 236-244P

[2]. LV Junjun, WU Guoqing, DU bo. Non-Gaussian under-water transient signals detection using Power-law detector. Acta Acustica, 2004, 29(4).

[3]. Wang Yan, Zou Nan,Fu Jin, Liang Guo-long. Transient Signal Detection Method Based on Partial Instantaneous Energy Density Level [J]. Journal of Electronics \& Information Technology,2013,35(7)

[4]. Xiong Xiao-Jun Yin Cheng Zhang Ba-i Lin DING Feng LI Da-Wei. Method Research of Forecasting Oil and Gas Using higher-order Statistics [J]. CHINESE JOURNAL OF GEOPHYSICS, 2004,47(5)

[5]. Zhang Guicai Shi Tielin Yang Shuzi. A Method for Extracting Mechanical Faults Features Based on Higher-Order Statistics [J]. J.Huazhong Univ. of Sci. \&Tech, 2004,47(5)

[6]. A.Nuttall. Detection performance of power-law processors for random signals of unknown location, structure, extent, and strength. NUWC-NPT Tech. Rep, Sept. 1994.

[7]. You-Ping Li, Wei-Qun Gan, Li Feng, Si-Ming Liu and A. Struminsky. The breakdown of the power-law frequency distributions for the hard X-ray peak count rates of solar flares [J]. Research in Astron. Astrophys. 2013 Vol. 13 No. 12, 1482-1492

[8]. HAN Jian-hui, YANG Ri-jie, WANG Wei. Research on power-law underwater acoustic transient signal detection based on wavelet transform [J]. Journal of System Simulation. 2008 Vol. 20 No. 13

[9]. Mallat S. Zero-Crossings of a wavelet transform[J].IEEE Transaction on information theory 1991,37 (4) :1019 1033

[10]. Xinhua Xu, Fu Xiao, Shengwei Wang.Enhanced chiller sensor fault detection,diagnosis and estimation using wavelet analysis and principal component analysis methods[J]. Applied Thermal Engineering 28 (2008) 226-237 